\title{
Micro-supercapacitors layer up for power
}

Nanoscale https://doi.org/10.1039/C9NR03917A (2019)

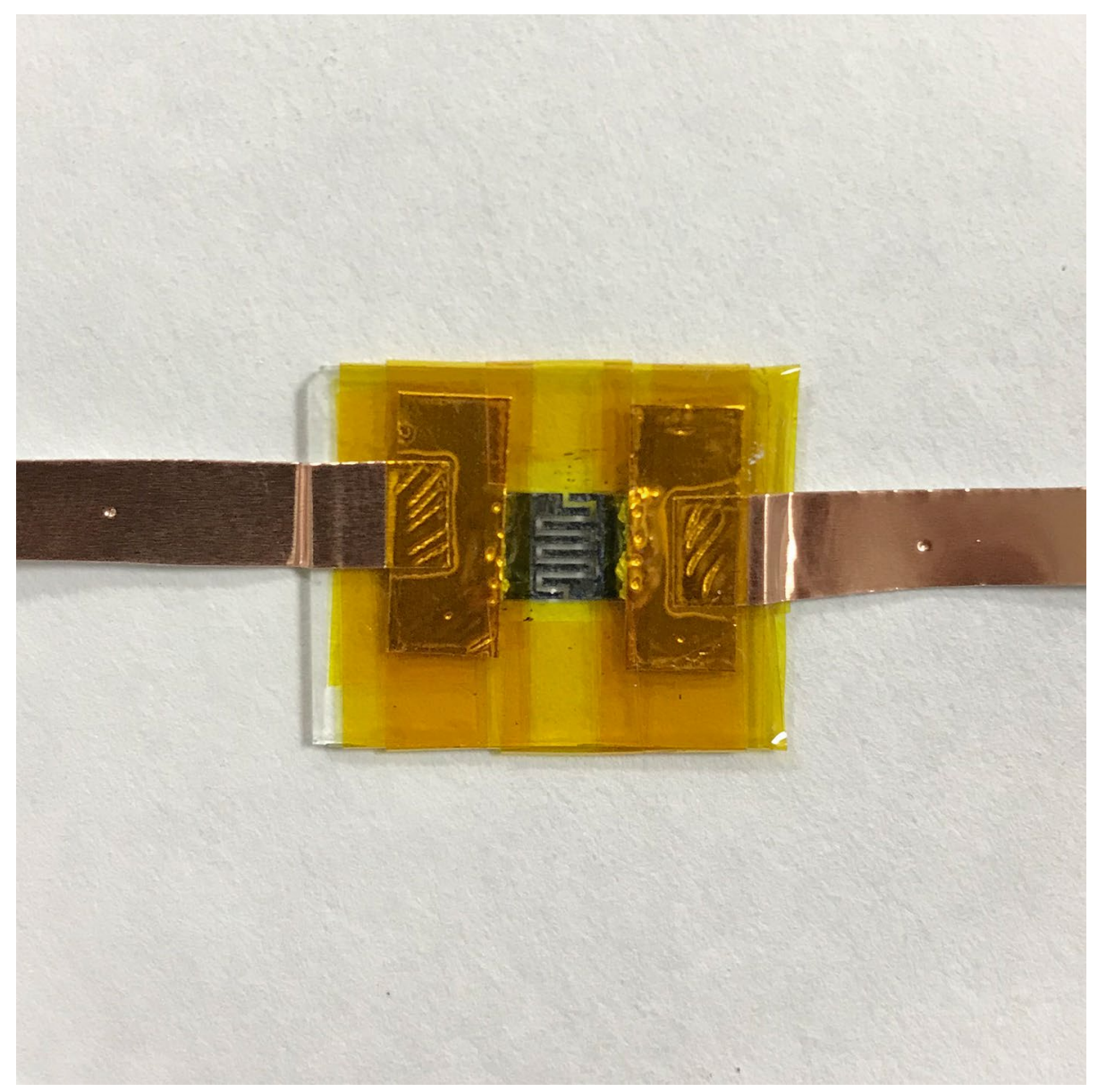

Credit: RSC

Micro-supercapacitors can be used as an alternative to batteries to store and release energy in nanoscale or wearable electronics, offering high power density and lower cost. However, the devices typically offer low areal capacitance, which limits their energy density, and low flexibility. Zhenxing Zhang, Erqing Xie and colleagues at Lanzhou University and Shenzhen University have now used highly flexible composite films to create micro-supercapacitors with high capacitance and flexibility.

The researchers fabricated the microsupercapacitors from individual or stacked layers, where each layer is a composite consisting of chemically reduced graphene and manganese oxide films. An electrolyte layer was added between the composite layers to bind each stack, and the microsupercapacitors were etched into the plane of this structure by laser engraving.
Bilayer micro-supercapacitors (consisting of composite-electrolyte-composite) exhibited an areal capacitance of 31.5 millifarads per centimetre squared, whereas a device containing five layers achieved an areal capacitance of 144.3 millifarads per centimetre squared. The bilayer device was also found to maintain its capacitance after 4,000 bending cycles. However, though adding layers provides a path to increase the areal capacity, and hence energy density, it was also found to lower power density (that is, the rate the stored energy can be released is lower). The power density was though still higher than that of thin-film lithium-ion batteries.

Matthew Parker

Published online: 15 August 2019

https://doi.org/10.1038/s41928-019-0297-z 